\title{
Input Power Factor Control of Matrix Converter Based Transmitter for Transient Electromagnetic Sounding
}

\author{
Gang Li
}

Key laboratory of geophysical exploration equipment, Ministry of Education (Jilin University) College of instrumentation and electrical engineering, Jilin University

\begin{abstract}
Three-phase to single-phase matrix converter (TSMC) is used as the transmitter for transient electromagnetic method (TEM) instrument, instead of an AC-DC converter and an H-bridge inverter. The transmitter produces excitation current for primary field. The output current has a waveform with a bipolar square of half duty cycle, which is required by TEM. The space vector PWM based on input currents is discussed in detail. The control method of input power factor is proposed for TSMC. The simulation model of TSMC is established and the simulations of different conditions are completed. The simulation results show the controllability of the input power factor is illustrated.
\end{abstract}

Key words - matrix converter; transient electromagnetic method; simulation; space vector modulation; power factor control

\section{Introduction}

With the wide application of power electronic converters, more and more instruments use switching power supply. In the geophysical exploration area, the electromagnetic method instrument has an important role [1-4]. The method is used in coal mine, metallic minerals, and geological mapping, etc. There are two kinds of electromagnetic method, time-domain and frequency-domain. The time-domain electromagnetic method is also called transient electromagnetic method (TEM). TEM is based on electromagnetic induction. The transmitter output current to the coils or the earth, to generate the primary field. When output current is off, the primary field rapidly disappears. Based on Lenz's law, the secondary field is generated in subsurface medium. The receiver of TEM instrument senses the secondary field. The data process system analyzes the electrical characteristics of the medium [5-6].

The process of TEM sounding is shown in Fig.1. Output current of the transmitter is shown in Fig.1 (a). It is also represent the primary field. Fig.1 (b) shows the secondary field of the abnormal body in the medium which is generated by turn-on and turn-off of the primary field. Because the primary field is much stronger than the secondary field, only the secondary field signal during the 
turn-off stage of the excitation current is sampling by the receiver, which is shown in Fig.1 (c).

The TEM instrument has two parts, transmitter and receiver with magnetic sensor. The topology of the conventional transmitter is shown in Fig.2, including electrical generator, AC/DC rectifier and $\mathrm{H}$ bridge inverter [7-9]. In order to quicken the turn-off speed of excitation current and reduce the influence of the remaining primary field, auxiliary circuit is needed in the conventional transmitter. In order to simplify the structure and control of TEM transmitter, three-phase to single-phase matrix converter (also called TSMC) is used as TEM transmitter. Compared with indirect AC-AC converters, such as $\mathrm{AC} / \mathrm{DC}$ rectifier and inverter using in conventional transmitter, TSMC has several advantages such as bidirectional power flow, high reliability, and compact design because of no bulky electrolytic capacitor or inductor in the dc link.

(a)

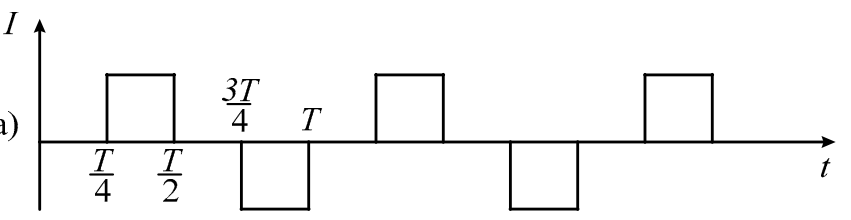

(b)

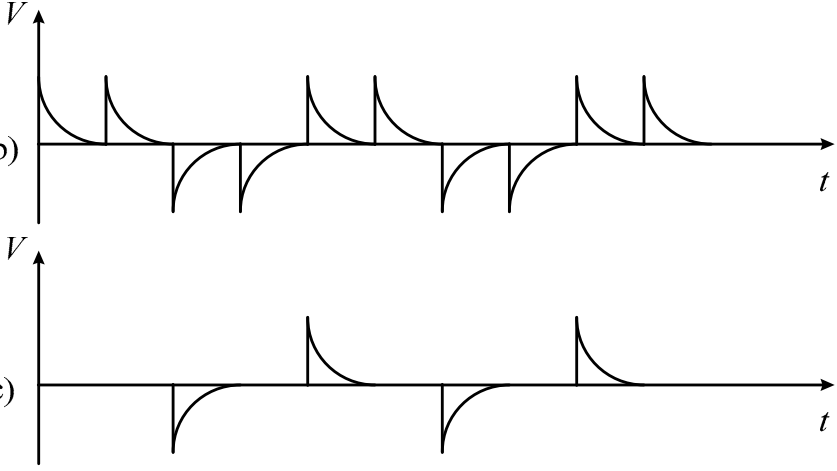

Fig.1 The process of TEM sounding

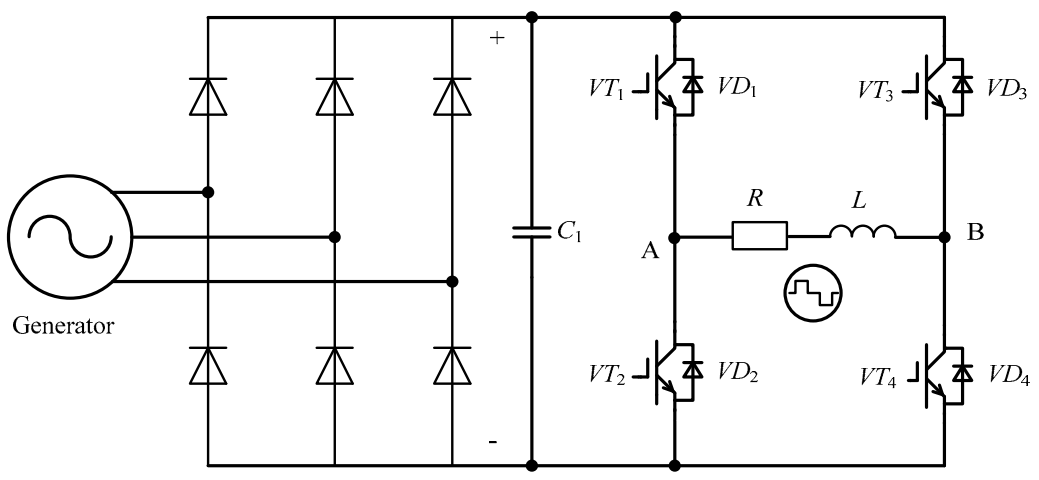

Fig.2 Topology of conventional TEM transmitter 


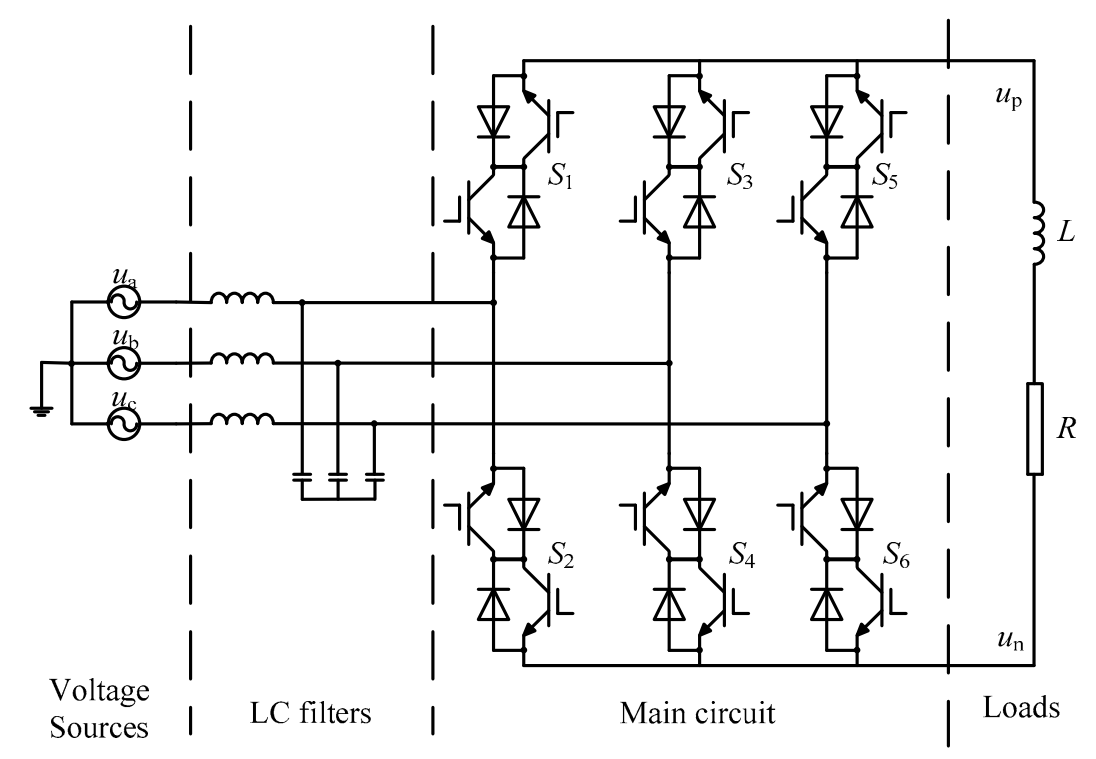

Fig.3 Topology of the proposed transmitter

In this paper, the PWM strategy for TSMC using in TEM is described in part II. The process of SVM is presents. The input power factor control for TSMC is present in part III. The impact of input LC filters is analyzed. Part IV gives the simulation model and shows the simulation results. Several simulations are implemented. Part V gives some conclusions.

\section{Space vector modulation for TSMC}

In the conventional transmitter of TEM, shown in Fig.2, the output current is controlled by an $\mathrm{H}$ bridge inverter. During the time $(\mathrm{T} / 4, \mathrm{~T} / 2)$, the DC voltage of $C_{1}$ will be output to the load. After the rising stage, the inductor $L$ will be saturated and the current will be decided by the input DC voltage and the resistor $R$. So the control method of $\mathrm{H}$ bridge inverter is very simple.An assumed three-phase symmetry system is as follows.

$$
\left\{\begin{array}{l}
u_{a}=V_{m} \cos \left(\omega_{\mathrm{i}} t\right) \\
u_{b}=V_{m} \cos \left(\omega_{\mathrm{i}} t-2 \pi / 3\right) \\
u_{c}=V_{m} \cos \left(\omega_{\mathrm{i}} t+2 \pi / 3\right)
\end{array}\right.
$$

According to the topology of a three-phase - Single - phase matrix converter, its input is a three-phase voltage source. The output is the resistive load, which is equivalent to the current source. Therefore, the input three-phase can not be short circuited, and the output side can not be open circuit. So the output voltage can only choose one of the input line-line voltages.

Because of the load resistance and inductance, the three-phase to single-phase matrix converter can control a current converter, and three-phase input currents can be expressed as, 


$$
\left\{\begin{array}{l}
i_{a}=S_{a} i_{d c} \\
i_{b}=S_{b} i_{d c} \\
i_{c}=S_{c} i_{d c}
\end{array}\right.
$$

The reference current space vector can be expressed as,

$$
\dot{\boldsymbol{I}}_{\text {ref }}=i_{\mathrm{a}}+i_{\mathrm{b}} \mathrm{e}^{j\left[\frac{2}{3} \pi\right.}+i_{\mathrm{c}} \mathrm{e}^{-j\left[\frac{2}{3} \pi\right.}
$$

From (2) and (3),

$$
\dot{\boldsymbol{I}}_{\text {ref }}=i_{\mathrm{dc}}\left(\mathrm{S}_{\mathrm{a}}+\mathrm{S}_{\mathrm{b}} \mathrm{e}^{j \llbracket \frac{2}{3} \pi}+\mathrm{S}_{\mathrm{c}} \mathrm{e}^{-j \square^{\frac{2}{3} \pi}}\right)
$$

The current space vector of a three-phase to single phase matrix converter is shown in Figure 4. $\boldsymbol{I}_{\text {ref }}$ is the reference current space vector, and $\theta$ is the phase angle. The times of each vector can be obtained from figure 6 .

$$
\left\{\begin{array}{l}
t_{1}=m T_{\mathrm{s}} \sin \left(\frac{1}{2} \pi-\theta\right) / \sin \left(\frac{1}{3} \pi\right) \\
t_{2}=m T_{\mathrm{s}} \sin \left(\theta-\frac{1}{6} \pi\right) / \sin \left(\frac{1}{3} \pi\right) \\
t_{0}=T_{\mathrm{s}}-t_{1}-t_{2}
\end{array}\right.
$$

Where $m$ is modulation index $(0<m \leq 0.866)$. And $T_{\mathrm{s}}$ is sampling period.

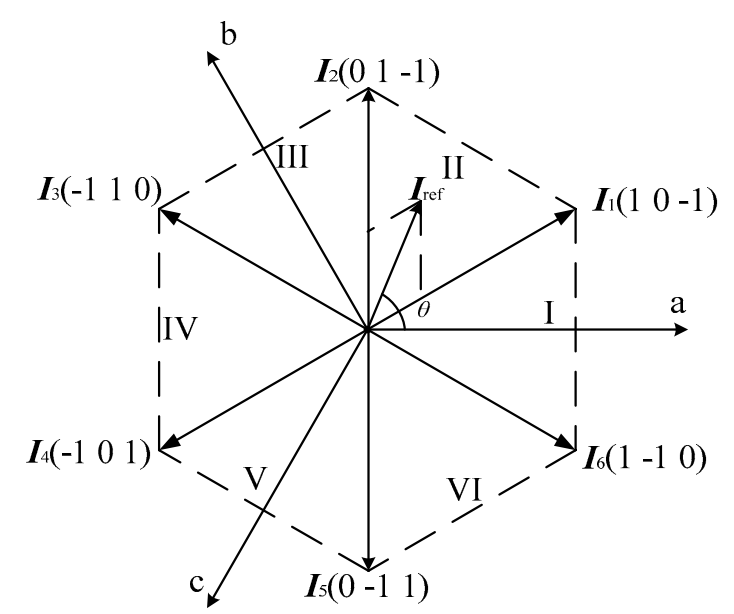

Fig.6 Current space vector of a three-phase to single phase matrix converter 


\section{Input power control of TSMC}

According to figure 6 , the relation between phase angle of input voltage and current vector sector is shown in Table I.

Table I the relation between phase angle of input voltage and current vector sector

\begin{tabular}{|c|c|}
\hline $\begin{array}{c}\text { Current vector } \\
\text { sector }\end{array}$ & $\begin{array}{c}\text { Phase angle of input voltage } \\
{[-\pi / 6, \pi / 6)}\end{array}$ \\
\hline 1 & {$[\pi / 6, \pi / 2)$} \\
\hline 2 & {$[\pi / 2,5 \pi / 6)$} \\
\hline 3 & {$[5 \pi / 6,7 \pi / 6)$} \\
\hline 4 & {$[7 \pi / 6,3 \pi / 2)$} \\
\hline 5 & {$[3 \pi / 2,11 \pi / 6)$} \\
\hline 6 & \\
\hline
\end{tabular}

The voltage sections of PWM correspond to the space vector sectors of SVM, shown in figure 7.

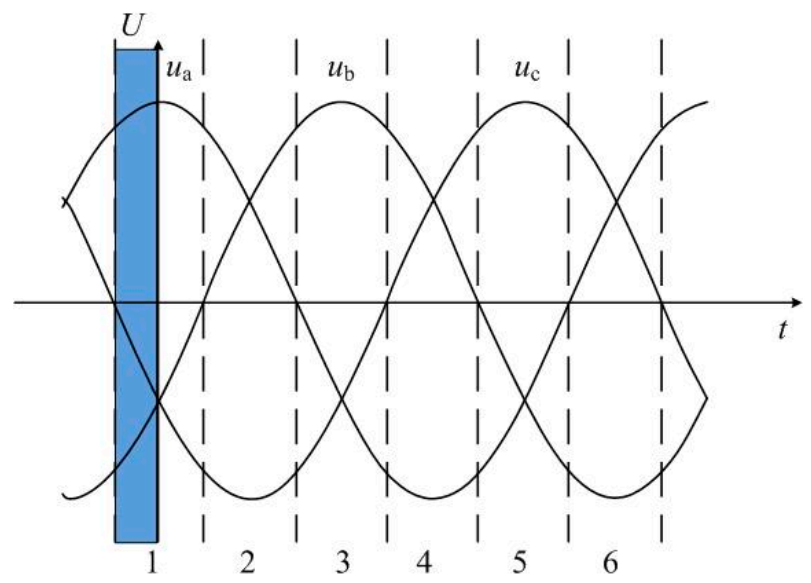

(a) input voltage sections

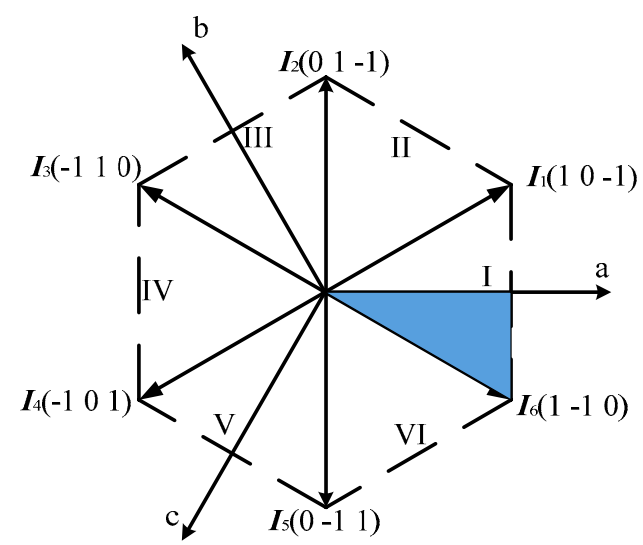

(b) sectors of input current space vector

Fig.7 the relation of input voltage sections and sectors of input current space vector

Therefore, as long as the phase of the input current reference vector of three-phase single-phase matrix converter is set, the phase difference between input current and input voltage can be adjusted, and the input power factor of matrix converter can be adjusted. Because of the input LC filters, the adjustment range of the input power factor is affected by the LC filter for the power grid side. Figure 8 is a simplified circuit of the input filter. 


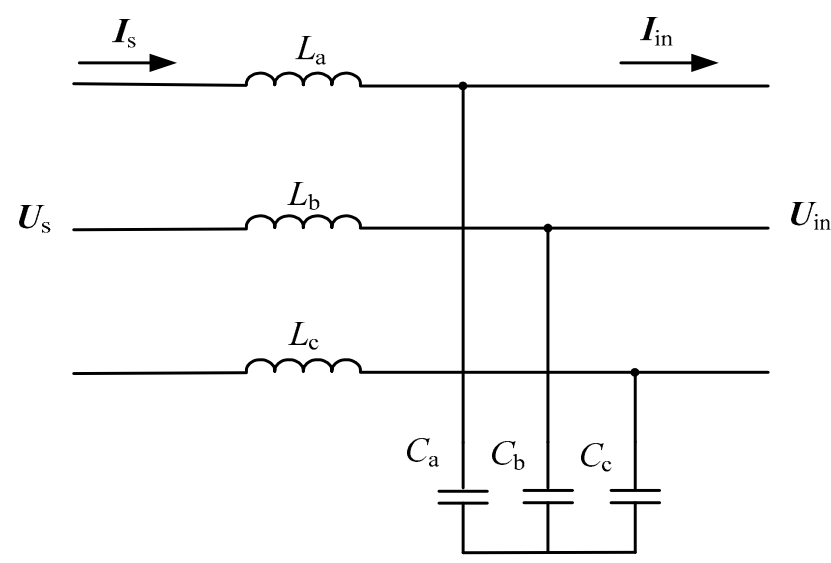

Fig. 8 simplified circuit of the input filter

$U_{\mathrm{s}}$ is input source voltage, and $\boldsymbol{I}_{\mathrm{s}}$ is input source current. $\boldsymbol{U}_{\text {in }}$ and $\boldsymbol{I}_{\text {in }}$ are input voltage and current of TSMC. The parameters of LC filters are, $L_{\mathrm{a}}=L_{\mathrm{b}}=L_{\mathrm{c}}=L=1 \mathrm{mH}$ and $C_{\mathrm{a}}=C_{\mathrm{b}}=C_{\mathrm{c}}=C=20 \mu \mathrm{F}$. The input voltage is defined as $\boldsymbol{U}_{\mathrm{s}}=U_{\mathrm{m}} \angle \alpha$. And input current of TSMC is $\quad \boldsymbol{I}_{\mathrm{in}}=I_{\mathrm{m}} \angle(\alpha-\varphi)$. And $\varphi$ is the setting phase difference.

$$
\left\{\begin{array}{l}
\boldsymbol{U}_{\mathrm{s}}-\boldsymbol{U}_{\mathrm{in}}=L \frac{\mathrm{d} \boldsymbol{I}_{\mathrm{s}}}{\mathrm{d} t} \\
\boldsymbol{I}_{\mathrm{s}}-\boldsymbol{I}_{\mathrm{in}}=C \frac{\mathrm{d} \boldsymbol{U}_{\mathrm{in}}}{\mathrm{d} t}
\end{array}\right.
$$

From (6)

$$
L C \frac{\mathrm{d}^{2} \boldsymbol{I}_{\mathrm{s}}}{\mathrm{d} t^{2}}+\boldsymbol{I}_{\mathrm{s}}-\left(\boldsymbol{I}_{\mathrm{in}}+C \frac{\mathrm{d} \boldsymbol{U}_{\mathrm{s}}}{\mathrm{d} t}\right)=0
$$

Considering fundamental component of input current in the steady state, the influence of the harmonic and the dynamic process is ignored. The input source current is defined as $\boldsymbol{I}_{\mathrm{s}}=I_{\mathrm{x}} \angle \beta$

$$
\begin{gathered}
I_{\mathrm{x}}\left(1-L C \omega_{\mathrm{i}}^{2}\right) \angle \beta=I_{\mathrm{m}} \angle(\alpha-\varphi)-C \omega_{\mathrm{i}} U_{\mathrm{m}} \angle\left(\alpha-\frac{1}{2} \pi\right) \\
\left\{\begin{array}{l}
I_{\mathrm{x}}=\frac{\sqrt{\left(I_{\mathrm{m}}\right)^{2}+\left(C \omega_{\mathrm{i}} U_{\mathrm{m}}\right)^{2}-2 C \omega_{\mathrm{i}} U_{\mathrm{m}} I_{\mathrm{m}} \sin \varphi}}{1-L C \omega_{\mathrm{i}}^{2}} \\
\beta=\alpha+\arctan \left(\frac{C \omega_{\mathrm{i}} U_{\mathrm{m}}-I_{\mathrm{m}} \sin \varphi}{I_{\mathrm{m}} \cos \varphi}\right)
\end{array}\right.
\end{gathered}
$$

The input power factor angle is related to the capacitance value of the LC filter, the amplitude of the load current and the set power factor angle. 


\section{Simulation results}

In order to verify the effectiveness of modulation algorithm based on three-phase to single-phase matrix converter, a simulation model is established, and the simulation parameters are shown in Table II.

Table II simulation parameters

\begin{tabular}{|l|l|}
\hline Parameters & Value \\
\hline RMS of input line-line voltage & $380 \mathrm{~V}$ \\
\hline Input source frequency & $50 \mathrm{~Hz}$ \\
\hline Load resistor $R$ & $15 \Omega$ \\
\hline Load inductor $L$ & $5 \mathrm{mH}$ \\
\hline Inductor of input filters $L_{f}$ & $1 \mathrm{mH}$ \\
\hline Capacitor of input filters $C_{f}$ & $20 \mu \mathrm{F}$ \\
\hline Sampling frequency & $10 \mathrm{kHz}$ \\
\hline
\end{tabular}

A bipolar square wave current signal with a ratio of $50 \%$ in figure 3 is a reference signal. The simulation waveforms with setting phase difference $\varphi=0,-\pi / 6$ and $\pi / 6$ are shown in figure $9-14$ respectively.

Because output current of TSMC is a bipolar square waveform with $50 \%$ duty cycle, input phase current is discontinuous sinusoidal waveform. When the setting phase difference $\varphi$ is 0 , input current is increasing with the input voltage synchronously with no oscillating process. When the setting phase difference $\varphi$ is not 0 , the input current is not synchronized with the voltage. Input current has an oscillating process.

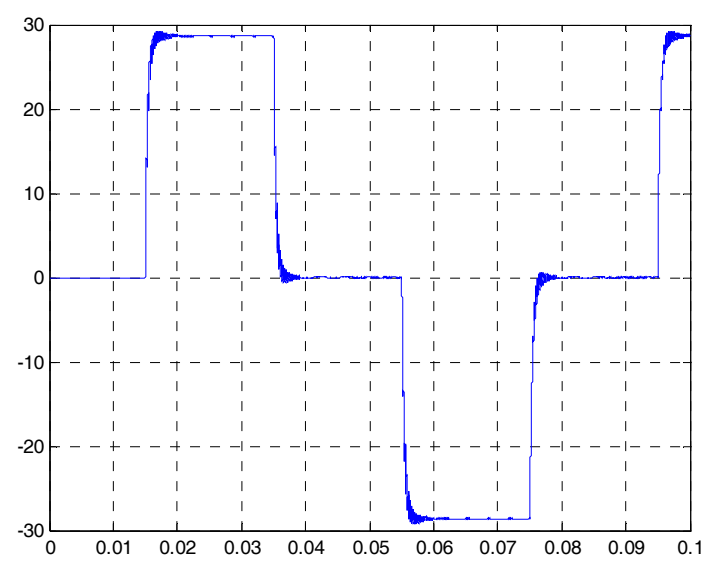

Fig.9 simulated output current of frequency $12.5 \mathrm{~Hz}$ with setting phase difference $\varphi=0$ 


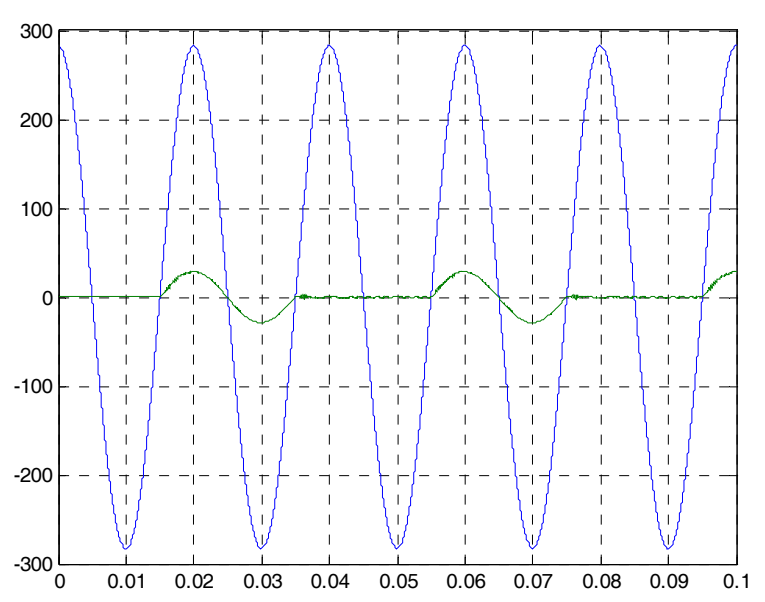

Fig.10 Simulated input phase voltage and current with setting phase difference $\varphi=0$

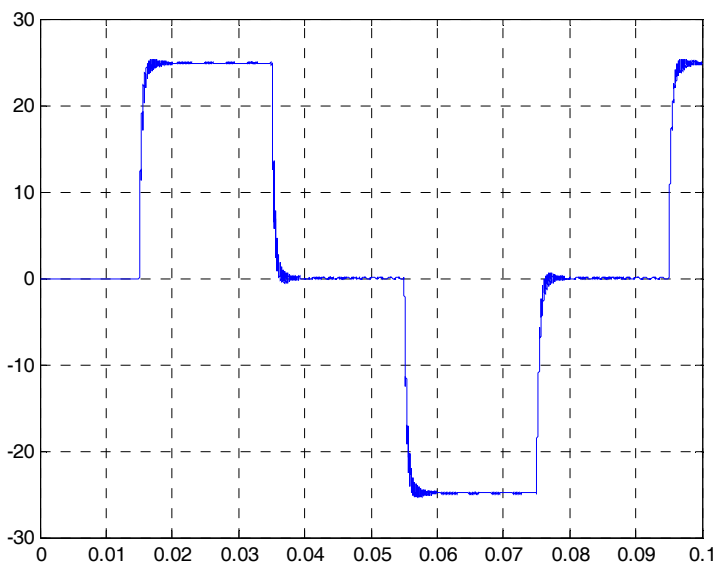

Fig.11 simulated output current of frequency $12.5 \mathrm{~Hz}$ with setting phase difference $\varphi=-\pi / 6$

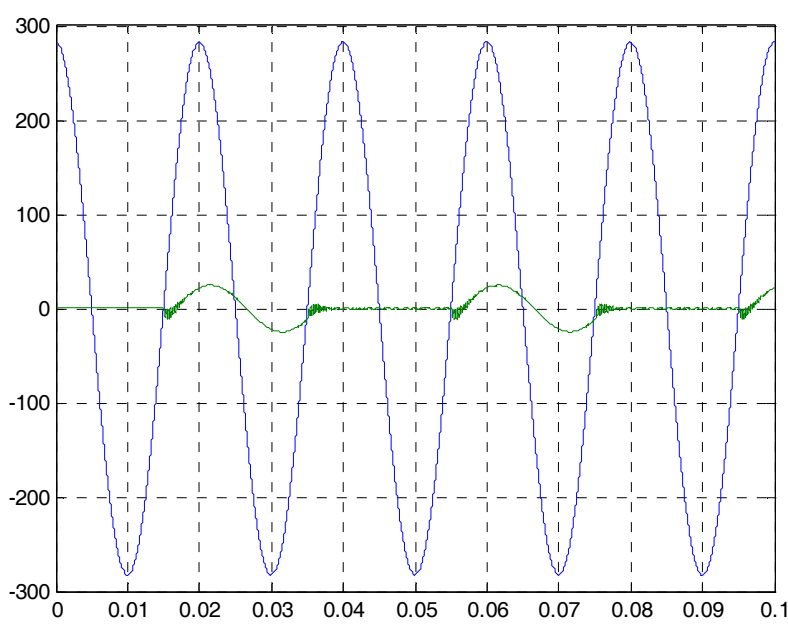

Fig.12 Simulated input phase voltage and current with setting phase difference $\varphi=-\pi / 6$ 


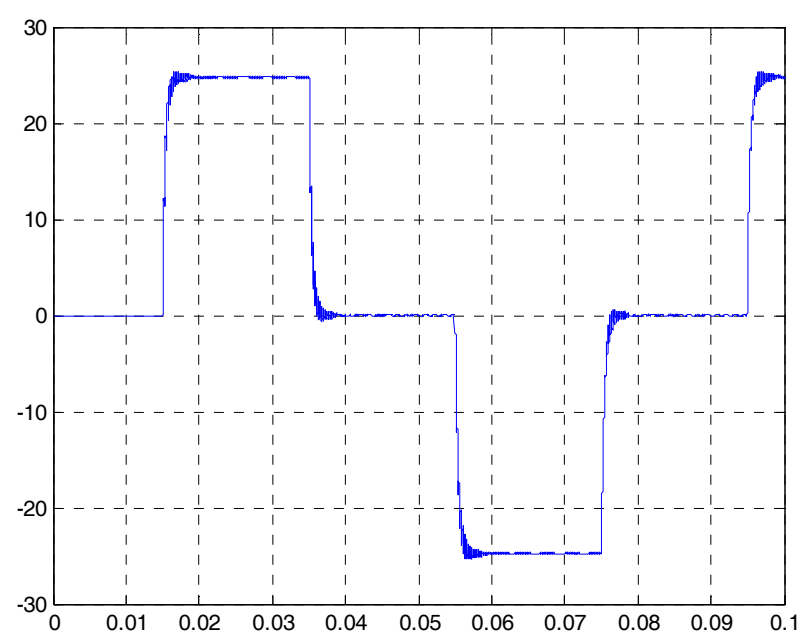

Fig.13 simulated output current of frequency $12.5 \mathrm{~Hz}$ with setting phase difference $\varphi=\pi / 6$

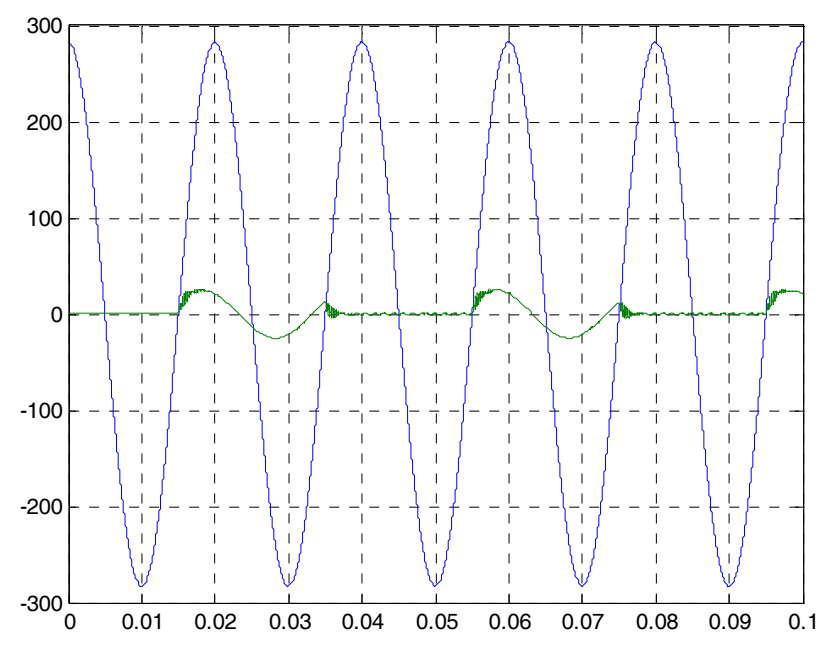

Fig.14 Simulated input phase voltage and current with setting phase difference $\varphi=\pi / 6$

\section{Conculsions}

In this paper, the working principle of transmitter based on three-phase to single phase matrix converter for TEM is studied. A simplified model and an equivalent circuit are established. The characteristics of transient electromagnetic current waveform are analyzed. The space vector PWM modulation algorithm based on input current is discussed. On this basis, the input power factor adjustment method of matrix converter is analyzed. A simulation model is established, and simulation experiments are carried out. The following conclusions are obtained.。

(1) The output current of three-phase to single-phase matrix converter is a bipolar square 
waveform with $50 \%$ duty cycle, not sine wave, which is different from the application of matrix converter in $\mathrm{AC}$ drive.

(2) The input power factor is adjusted by setting the reference phase angle of the input current space vector, but the parameters such as the load current and the LC filter have influence on it.

(3) The simulation results verify the effectiveness of the modulation algorithm and the power factor adjustment method for three-phase to single-phase matrix converter.

\section{Acknowledgement}

The authors would like to thank National Natural Science Foundation of China (No. 51507073) for their supports in our work.

\section{References}

[1] P. A. Reninger, G. Martelet, E. Lasseur, L. Beccaletto, J. Deparis, J. Perrin, and Y. Chen, "Geological environment of karst within chalk using airborne time domain electromagnetic data cross-interpreted with boreholes,” J. Appl. Geophy., vol. 106, pp. 173-186, May. 2014.

[2] H. El-Kaliouby, and O. Abdalla, "Application of time-domain electromagnetic method in mapping saltwater instrusion of a coastal alluvial acquifer, North Oman," J. Appl. Geophy., vol. 115, pp. 59-64, Feb. 2015.

[3] M. I. Epov, E. Y. Antonov, N. N. Nevedrova, V. V. Olenchenko, E. V. Pospeeva, D. V. Napreev, A. M. Sanchaa, V. V. Potapov, and A. E. Plotinkov, "Integrated electromagnetic and geochemical surveys for petroleum exploration in West Siberia," Russ. Geo. Geophy., vol. 55, no. 5-6, pp.763-774, May - Jun. 2014.

[4] N. N. Nevedrova, E. V. Deev, and A. M. Sanchaa, "Deep structure and margins of the Kurai Basin (Gorny Altai), from controlled-source resistivity data," Russ. Geo. Geophy., vol. 55, no. 1, pp. 98-107, Jan. 2014.

[5] P. T. Simard, R. Chesnaux, A. Rouleau, R. Daigneault, P. A. Cousineau, D. W. Roy, M. Lambert, B. Poirier, and L. Poignant-Molina, "Imaging quaternary glacial deposits and basement topography using the transient electromagnetic method for modeling acquifer environments," $J$. Appl. Geophy., vol.119, pp. 36-50, Aug. 2015.

[6] A. E. Plotnikov, "Evaluation of limitations of the transient electromagnetic method in 
shallow-depth studies: numerical experiment," Russ. Geo. Geophy., vol. 55, no.7, pp. 907-914, Jul. 2014.

[7] Kaichang Xue, Fengdao Zhou, Shuang Wang, and Jun Lin, "Constant-current control method of multi-function electromagnetic transmitter,” Rev. Sci. Instru., vol. 86, no. 2, pp. 024501-1 10, Feb. 2015.

[8] Kaichang Xue, Shuang Wang, Jun Lin, Gang Li, and Fengdao Zhou, "Loss analysis and air-cooled design for a cascaded electrical source transmitter," J. Power Electron., vol. 15, no.2, pp. 530-543, Feb. 2015.

[9] S. A. Allah, T. Mogi, H. Ito, A. Jomori, Y. Yuuki, E. Fomenko, K. Kiho, H. Kaieda, K. Suzuki, and K. Tsukuda, "Three-dimensional resistivity characterization of a coastal area: application of grounded electrical-source airborne transient electromagnetic (GREATEM) survey data from Kujukuri Beach, Japan,” J. Appl. Geophy., vol. 99, pp. 1-11, Sep. 2013. 